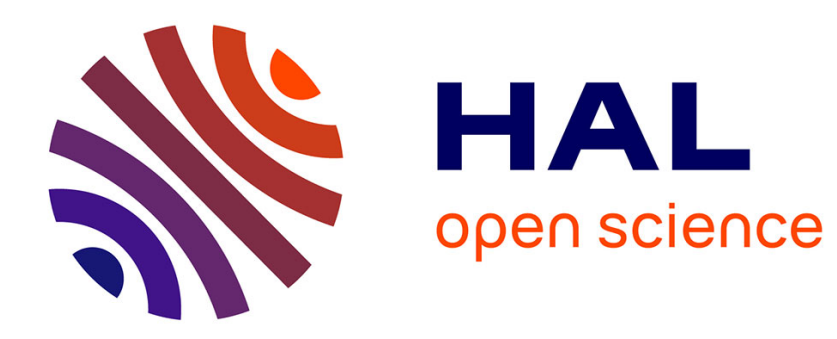

\title{
La reconnaissance des sols historiques urbains par méthodes géophysiques
}

\author{
Cédric Panissod, Michel Dabas
}

\section{To cite this version:}

Cédric Panissod, Michel Dabas. La reconnaissance des sols historiques urbains par méthodes géophysiques. Histoire et mesure, 1999, XIV (3/4), pp.221-248. 10.3406/hism.1999.1510 . hal-02925641

\section{HAL Id: hal-02925641 \\ https://hal.science/hal-02925641}

Submitted on 30 Aug 2020

HAL is a multi-disciplinary open access archive for the deposit and dissemination of scientific research documents, whether they are published or not. The documents may come from teaching and research institutions in France or abroad, or from public or private research centers.
L'archive ouverte pluridisciplinaire HAL, est destinée au dépôt et à la diffusion de documents scientifiques de niveau recherche, publiés ou non, émanant des établissements d'enseignement et de recherche français ou étrangers, des laboratoires publics ou privés. 


\section{La reconnaissance des sols historiques urbains par méthodes} géophysiques

Michel Dabas, Cédric Panissod

\section{Citer ce document / Cite this document :}

Dabas Michel, Panissod Cédric. La reconnaissance des sols historiques urbains par méthodes géophysiques. In: Histoire \& Mesure, 1999 volume $14-n^{\circ} 3-4$. Habitat et espace urbain. pp. 221-247;

doi : 10.3406/hism.1999.1510

http://www.persee.fr/doc/hism_0982-1783_1999_num_14_3_1510

Document généré le 14/06/2016 


\title{
Resumen
}

histoire urbaine, archéologie

\begin{abstract}
The Study of Historical Urban Soils with Geophysical Methods

The study of the urban soil structure is nowadays an important preoccupation for environmental, historical and technical reasons. These grounds are characterized by their important complexity, which makes a pluridisciplinary study necessary. Geophysical methods allow to obtain nondestructive information about these soils. However, the urban context (overlapped 3D features, limited accessible spaces, electromagnetic perturbations) significantly limits the field of the methods that can be used for this type of investigation. A measurement protocol associating ground penetrating radar and the electrostatic method, was defined for the survey and the evaluation of the heritage present in historical urban soils. In this paper, we expose some case studies: study of ancient buildings under present buildings (the cathedral of Gerona, Spain), study of fortifications (La Rochelle, France) and detection of ancient roads (the Heptastadium of Alexandria, Egypt).
\end{abstract}

\section{Résumé}

La reconnaissance de la structure des sols urbains est aujourd'hui une préoccupation majeure pour des raisons environnementales, historiques et techniques. Ces sols sont caractérisés par leur grande complexité structurelle qui rend nécessaire une approche pluridisciplinaire. Les méthodes géophysiques permettent d'acquérir des informations de façon non destructive. Cependant, le contexte urbain (structures 3D superposées, espaces accessibles limités, perturbations électromagnétiques) limite considérablement le champ des méthodes adaptables à ce type de reconnaissance. Un protocole de mesure associant le radar-sol et la méthode électrostatique a été défini pour la reconnaissance et l'évaluation du patrimoine présent sur les sites historiques urbains. Nous présentons quelques exemples des résultats obtenus : étude de bâtis anciens sous les édifices actuels (la cathédrale de Gérone, Espagne), étude de fortifications (La Rochelle, France) et recherche de voies anciennes (l'Heptastade d'Alexandrie, Égypte). 
Histoire \& Mesure, 1999, XIV-3/4, 221-248

\title{
Cédric Panissod \& Michel Dabas*
}

\section{La reconnaissance des sols historiques urbains par méthodes géophysiques}

\begin{abstract}
Résumé. La reconnaissance de la structure des sols urbains est aujourd hui une préoccupation majeure pour des raisons environnementales, historiques et techniques. Ces sols sont caractérisés par leur grande complexité structurelle qui rend nécessaire une approche pluridisciplinairc. Les méthodes géophysiques permettent dacquérir des informations de façon non destructive. Cependant, le contexte urbain (structures 3I) superposées, espaces accessibles limités, perturbations électromagnétiques) limite considérablement le champ des méthodes adaptables à ce type de reconnaissance. Un protocole de mesure associant le radar-sol et la méthode électrostatique a été défini pour la reconnaissance et l'évaluation du patrimoine présent sur les sites historiques urbains. Nous présentons quelques exemples des résultats obienus : étude de bâtis anciens sous les édifices actuels (la cathédrale de Gérone, Espagne), étude de fortifications (La Rochelle, France) et recherche de voies anciennes (l'Heptastade d'Alexandrie, Égypte).
\end{abstract}

Abstract. The Study of Historical Urban Soils with Geophysical Methods. The study of the urban soil structure is nowadays an important preoccupation for environmental, historical and technical reasons. These grounds are characterized by their important complexity, which makes a pluridisciplinary study necessary. Geophysical methods allow to obtain nondestructive information about these soils. However, the urban context (overlapped 3D features, limited accessible spaces, electromagnetic perturbations) significantly limits the ficld of the methods that can be used for this type of investigation. A measurement protocol associating ground penetrating radar and the electrostatic method, was defined for the survey and the evaluation of the heritage present in historical urban soils. In this paper, we expose some case studies: study of ancient buildings under present buildings (the cathedral of Gerona, Spain), study of fortifications (La Rochelle, France) and detection of ancient roads (the Heptastadium of Alexandria, Egypt).

La reconnaissance de la structure des sols urbains est aujourd'hui une préoccupation majeure de chercheurs de spécialités très variées pour des raisons environnementales, historiques, techniques, etc. On assiste, en effet, d'une part, à une extension du domaine constitué par les sols urbains avec le développement des zones péri-urbaines, et d'autre part, à des réaménagements du centre des villes actuelles qui sont rendus nécessaires

\footnotetext{
* Département de (jéophysique Appliquéc, U.M.R. 7619 Sisyphe, Université Pierre et Maric Curie/Cins, 4, place Jussicu, 75252 Paris Cedex 05 . e-mail : cpanissod $a$ ccr.jussicu.fr.
} 
par l'intensification de leur utilisation et le respect de nouvelles normes. On pense notamment aux réseaux d'assainissement de nombreuses agglomérations françaises qui devraient faire l'objet de rénovation dans les prochaines années. Une bonne connaissance historique de ces réseaux est indispensable tant du point de vue des techniques de construction que de celui de leur mise en place. C'est dans ce contexte que les relations qui doivent exister entre les concessionnaires, les ingénieurs en génie civil, les archéologues et les historiens prennent toute leur importance.

La complexité des sols rend nécessaire une approche pluridisciplinaire de leur structure, bien que les préoccupations de chacun des intervenants présentent parfois des aspects contradictoires. Les méthodes géophysiques permettent d'acquérir de façon non destructive une information originale sur ces sols, et de proposer un premier schéma de leur structure en mettant en évidence des hétérogénéités qui se traduisent par des variations de paramètres physiques.

Dans cet article, on a cherché, dans un premier temps, à préciser la notion de sol urbain et, ce, en tenant compte de la diversité des approches disciplinaires. Dans un second temps, ont été décrites les méthodes géophysiques utilisées pour l'investigation de ces sols. Enfin, après avoir évoqué les enjeux et les problématiques auxquels ces reconnaissances peuvent apporter des éléments de réponse, ont été présentés trois exemples de prospections géophysiques en milieu urbain.

\section{Notion de sol urbain}

Le sol ou sous-sol urbain est un espace de vie, un espace partagé. Il s'agit d'un espace de structure complexe, qui peut évoluer dans un temps très court, et pour lequel une intervention à un endroit donné peut avoir des conséquences sur un autre endroit sćparé par une distance relativement importante :

- pour l'archéologue : le sol urbain est un ensemble de milieux et de surfaces les délimitant, dans et sur lesquels les habitants ont construit, agi et détruit... C'est un milieu hétérogène et «culturel», dont le contenu révèle l'histoire de la ville : c'est une archive;

- pour l'ingénieur en génie civil: le sol urbain est à la fois un support et un contenant d'ouvrages. C'est un environnement dont les propriétés géotechniques sont très variables et qui est caractérisé par une forte densité de constructions et de réseaux ;

- pour le géophysicien : le sol urbain est un milieu essentiellement constitué de structures en trois dimensions (3D) d'origine anthropique et soumis aux actions permanentes de l'homme et aux circulations d'eau chargée de différents polluants. La nature des matériaux rencontrés est 
suffisamment diverse et contrastée pour que ceux-ci puissent être mis en évidence par les méthodes géophysiques. Une difficulté majeure provient en revanche de la superposition des informations et de leur concentration dans de petits espaces. Enfin, une autre difficulté provient des perturbations des signaux géophysiques associées à l'environnement urbain (vibrations, signaux électromagnétiques, courants parasites, etc.).

Cette première constatation de l'existence d'une division du problème des sols urbains par « spécialités » conduit, paradoxalement, à rapprocher ces différentes disciplines ; c'est en particulier le cas pour les problèmes de reconnaissance, dans la mesure où il s'agit, le plus souvent, de mettre en évidence les variations de paramètres physiques caractéristiques du sous-sol, et de les corréler à l'histoire de ces sols urbains.

\section{Méthodes}

L'expérience acquise depuis plusieurs dizaines d'années par la prospection géophysique appliquée à l'archéologie ', ainsi que l'apparition de nouvelles méthodes de mesure (en particulier le radar-sol dont on a très vite démontré l'intérêt pour la détection des réseaux en génie civil) ont contribué au développement des prospections archéologiques en milieu urbain. L'utilisation de méthodes non destructives est un atout considérable dans ce milieu où toute intervention classique (tranchée, forage, ...) a des conséquences sociales et économiques non négligeables. Cependant, les spécificités propres aux zones urbaines (sols construits, lignes électriques, éléments métalliques, etc.) viennent limiter le champ d'application des méthodes normalement utilisées en zone rurale. Nous nous limiterons ici à l'évocation du principe de ces méthodes et de leurs conditions d'application au milieu urbain, en renvoyant le lecteur aux ouvrages qui viennent d'être cités pour de plus amples détails.

\section{Méthodes peu adaptées aux milieux urbains}

\section{Méthode électrique}

Nous commençons par la méthode électrique qui est aujourd'hui la méthode la plus couramment utilisée pour la prospection archéologique en milieu rural et qui reste la méthode de base en génie civil. Le principe de cette méthode consiste à utiliser quatre électrodes métalliques avec lesquelles on établit un contact galvanique avec le sol. Deux électrodes permettent l'injection d'un courant continu dans le sol et les deux autres

1. C. A. HISSE: et al., 1986 ; I. S(OH.IAR el al. 1990. 
permettent la mesure d'une différence de potentiel qui s'établit en fonction des hétérogénéités du terrain. La résistivité apparente est proportionnelle au rapport de cette différence de potenticl à l'intensité du courant injecté et dépend de la géométrie du dispositif employé. Nous reviendrons sur ces notions avec la méthode électrostatique qui n'a pas besoin de contact galvanique : en effet, le principal problème de la méthode électrique en milieu urbain correspond à l'impossibilité de planter les électrodes sur les surfaces construites. Cette méthode est donc limitée à l'exploration de jardins, de terrains de sport et d'autres espaces dont la surface n'est ni bitumée, ni cimentée.

\section{Méthodes électromagnétiques à basses fréquences $(<I M H z$.)}

Ces méthodes (dont la méthode dite «Slingram» du nom de son inventeur) permettent de mesurer la conductivité électrique apparente (inverse de la résistivité apparente) du sol. Dans ce cas, la circulation du courant électrique dans le sol n'est plus obtenue à travers des électrodes mais en faisant varier le flux du champ magnétique créé par un dipôle magnétique constitué d'une petite bobine parcourue par un courant. Cette méthode est particulièrement sensible à la présence de lignes électriques et d'objets métalliques, ce qui rend son utilisation souvent problématique dans les milieux urbanisés.

\section{Méthode magnétique}

Les mesures magnétiques (champ total ou gradient) sont également très courantes en prospection archéologique. Elles permettent de mettre en évidence, avec une précision aujourd'hui bien inférieure à ln $T$, des anomalies du champ magnétique terrestre qui ont pour origine des contrastes d'aimantation dans les sols. Néanmoins, ces mesures sont difficilement envisageables en milieu urbain du fait de la présence inévitable de corps perturbateurs (fer, acier) dans le voisinage et de courants électriques parasites.

\section{Méthode sismique}

La méthode sismique consiste à émettre des ondes acoustiques dans le sol au moyen de sources vibrantes (ou de marteaux, d'explosifs...) et à étudier les ondes réfléchies (sismique-réflexion) ou réfractées (sismiqueréfraction) par les interfaces du milieu (surface séparant deux corps où les vitesses des ondes sismiques diffèrent). Cette méthode est très lourde à mettre en ouvre : l'installation des capteurs impose en effet de créer avec le sol une liaison mécanique, qui présente la même difficulté que l'utilisation d'électrodes. Elle est, par ailleurs, sensible aux vibrations émises, notamment par le trafic routier, et il n'est pas simple de disposer d'une source suffisamment puissante pour s'affranchir de cette gêne. 


\section{Méthode gravimétrique}

La méthode gravimétrique permet de mettre en évidence les anomalies du champ de pesanteur terrestre, qui ont pour origine les contrastes de densité des matériaux constituant le sous-sol. Elle est en particulier très efficace pour la détection de vides comme les hypogées, les caves, les carrières, etc. Néanmoins, la précision requise par ce type de mesure, son coût, ainsi que le nombre de mesures nécessaires pour échantillonner de façon satisfaisante les anomalies en prospection de sub-surface deviennent rapidement un obstacle à sa mise en ouvre.

\section{Méthodes bien adaptées aux milieux urbains}

On dispose finalement de deux méthodes géophysiques bien adaptées au contexte urbain :

- la méthode radar (envoi d'une onde électromagnétique haute fréquence dans le sol et étude de ses réflexions sur les différentes interfaces situées en profondeur);

- la méthode électrostatique (mesure de la résistivité apparente).

\section{Méthode radar-sol (Ground Penetrating Radar ou GPR)}

Le radar-sol est constitué de deux antennes ( $C f$. fig. la) qui vont être déplacées simultanément le long d'un profil. L'une des antennes (dite émettrice) émet une onde électromagnétique haute fréquence (entre 100 et $1000 \mathrm{MHz}$ ) qui est réfféchie à l'interface de deux milieux dont les constantes diélectriques $\varepsilon_{1}$ et $\varepsilon_{2}$ diffèrent. La profondeur d'investigation de cette méthode dépend de la fréquence de l'onde émise et de la nature du sous-sol. Le coefficient de réflexion :

$$
R_{12}=\frac{\sqrt{\varepsilon_{2}}-\sqrt{\varepsilon_{1}}}{\sqrt{\varepsilon_{1}}+\sqrt{\varepsilon_{2}}}
$$

régit l'amplitude (en énergie) de l'onde renvoyée vers la surface. Cette onde est alors reçue par la deuxième antenne dite réceptrice ( $C f$. fig. 2 ). Le déplacement régulier le long d'un profil de l'ensemble formé par les deux antennes permet d'obtenir une sorte de coupe verticale du terrain $(C f$. fig. 3). On y trouve, en abscisse, la distance parcourue le long du profil et, en ordonnée, le temps mis par l'onde électromagnétique pour parcourir le chemin entre l'antenne émettrice et l'antenne réceptrice. Dans le cas de l'onde réfléchie qui nous intéresse, il s'agira d'un temps double correspondant au trajet aller (émetteur-interface) et retour (interface-récepteur) de l'onde. L'interface entre deux couches horizontales se traduira sur cette coupe par une limite horizontale; de même dans le cas de couches en pente, on pourra observer des interfaces obliques sur le graphique, mais dont les pentes ne seront pas, pour des raisons d'échelle, les pentes réelles. 
En revanche, des structures complexes seront détectées sous la forme de réflexions encore plus complexes et difficiles à interpréter sur la coupe brute. Ces coupes devront, alors, être traitées selon un processus proche de celui utilisé en traitement sismique pour les applications pétrolières.

Figure 1. Appareils de mesure géophysique adaptés à la prospection sur des sols urbains

a. Déplacement du couple d'antennes du radar-sol dans la cathédrale de Varsovie

b. Déplacement des pôles électrostatiques constitués de plaques conductrices souples sur une chaussée d'Alexandrie

c. Prototype opérationnel de multipôle électrostatique urbain
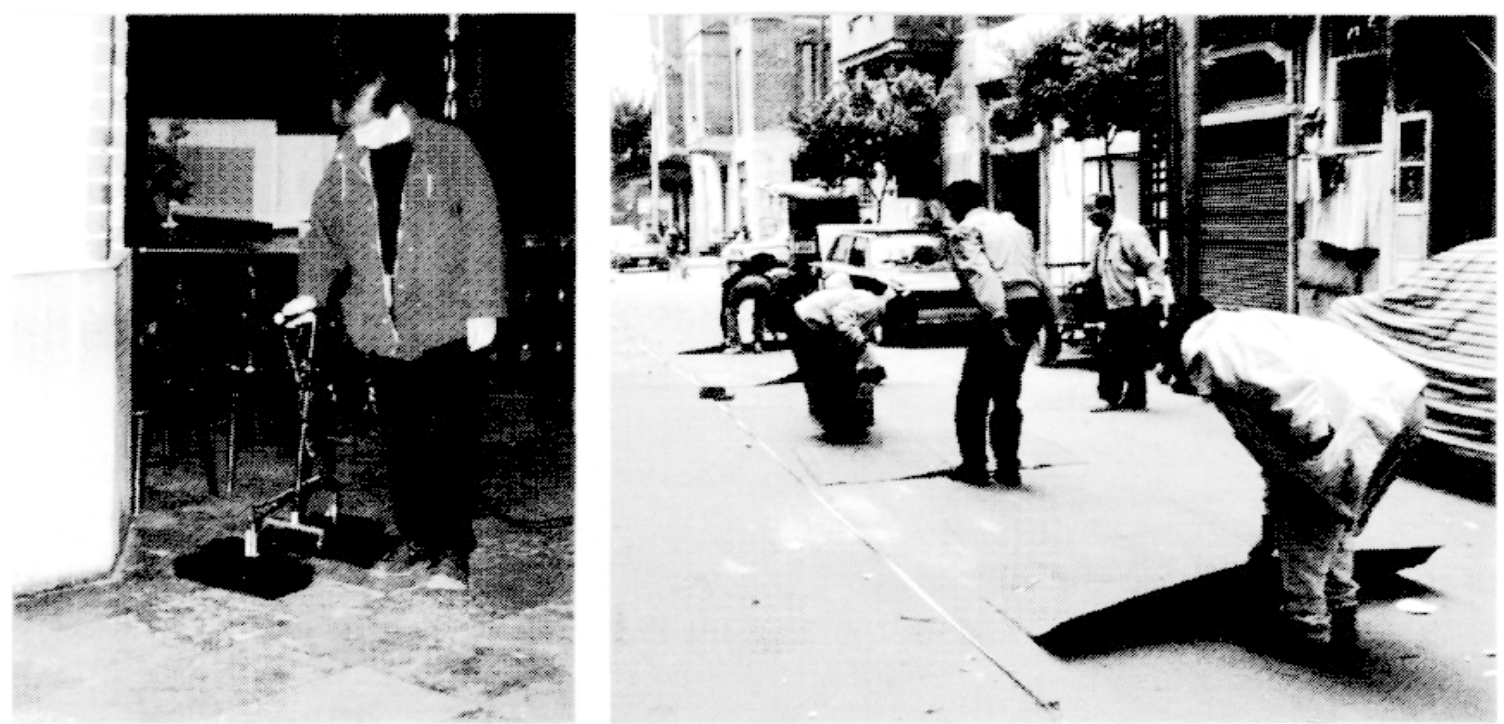

a

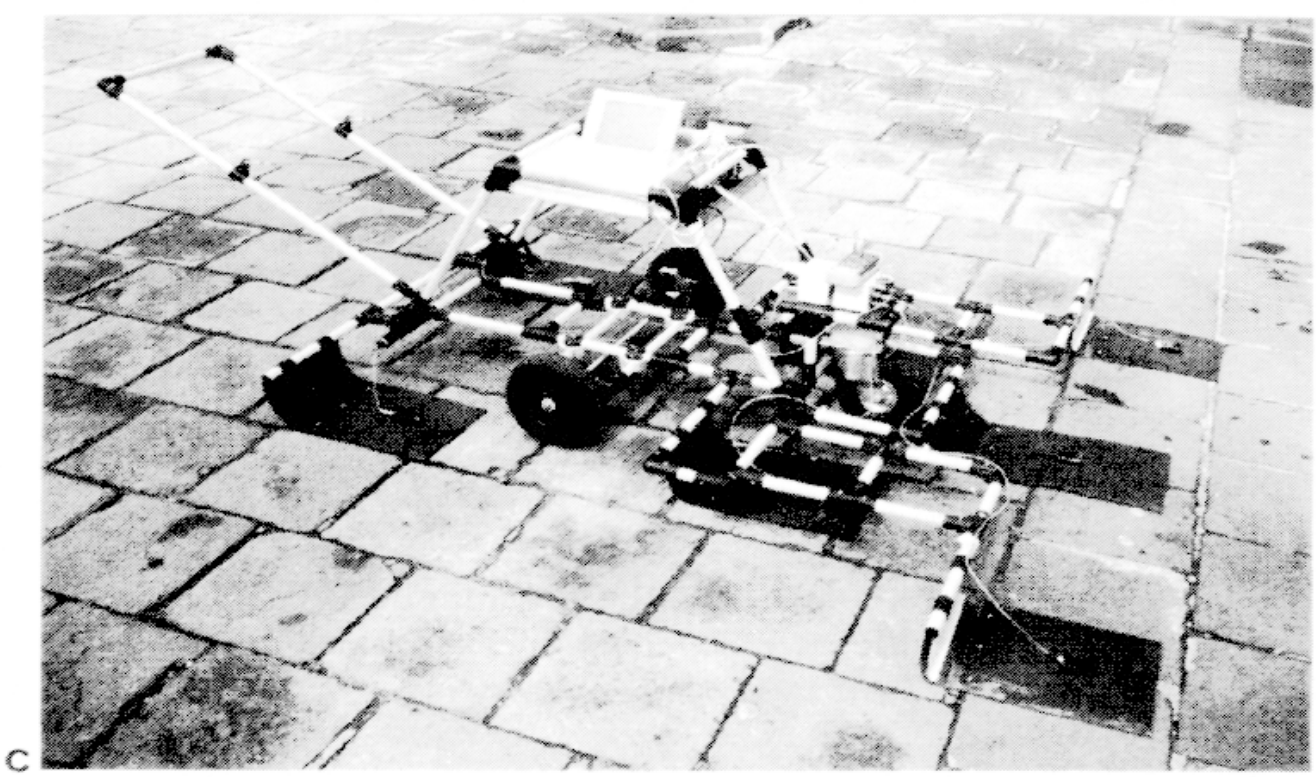

Les pôles sont constitués de plaques conductrices souples posées sur le sol; les instruments de mesure sont posés sur l'appareil qui peut être déplacé par traction manuelle, à gauche. 
Figure 2. Schéma de la mise en ceuvre d'une prospection radar-sol

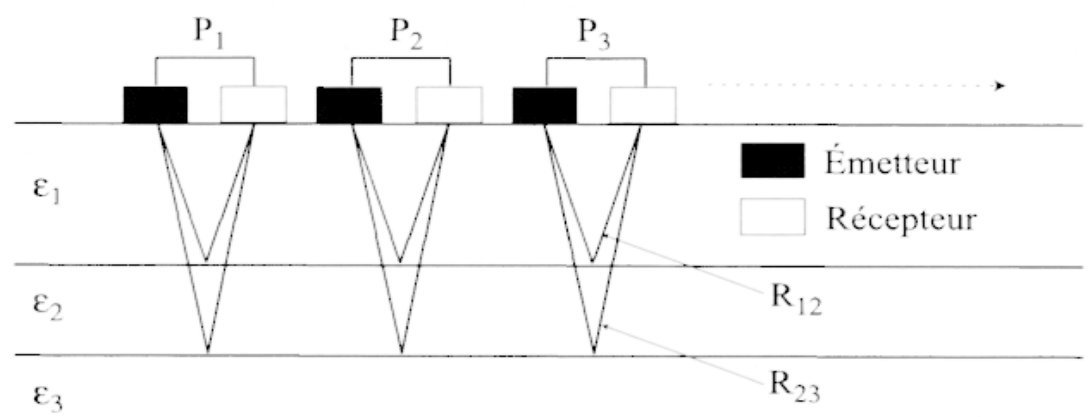

En $\mathrm{P}_{1}, \mathrm{P}_{2}$ et $\mathrm{P}_{3}$, sont représentées des positions successives des antennes le long d'un profil. $\mathrm{R}_{12}$ est la réflexion sur une première interface rencontrée entre un milieu 1 et un milieu 2 (voir texte); $\mathrm{R}_{23}$ est la réflexion sur une éventuelle interface plus profonde.

Figure 3. Section radar réalisée dans l'abbaye Saint-Germain d'Auxerre

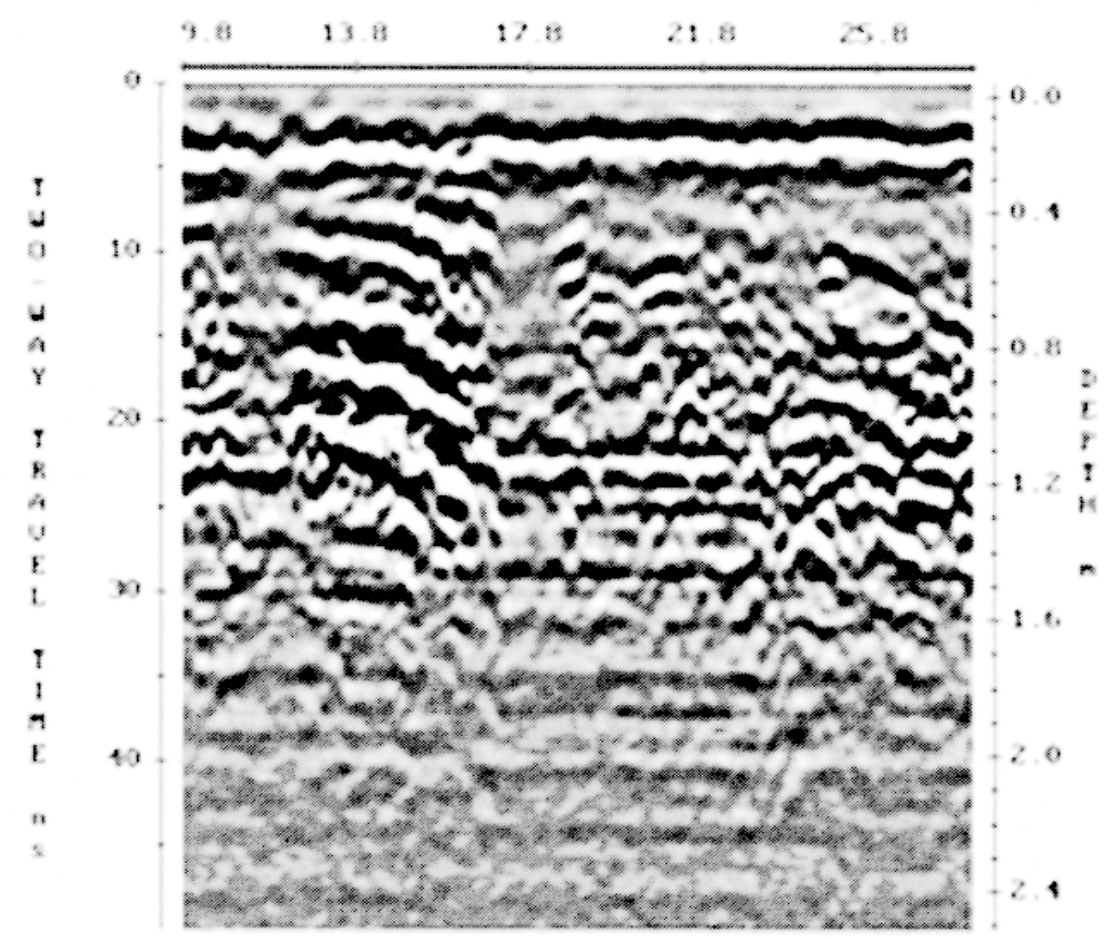

Chaque trace verticale correspond à une position des antennes repérée en mètres sur l'échelle horizontale ; sur chaque trace, le marquage en noir indique le dépassement d'un seuil d'énergie de la réponse des interfaces rencontrés à différents intervalles de temps repérés sur l'échelle verticale (voir fig. 2).

Cependant, ce type de coupe ne répond que partiellement aux attentes des problématiques archéologiques pour lesquelles les structures doivent souvent être en trois dimensions, et pour lesquelles la cohérence entre 
plusieurs coupes réalisées parallèlement et selon un échantillonnage précis, constitue un indice de fiabilité de réponse plus fort pour l'interprétation. Aussi des algorithmes ont été développés pour obtenir, à partir de ces coupes acquises à la surface selon un maillage de mesure fin dans deux directions $\mathrm{X}$ et $\mathrm{Y}$, des cartes en «tranches de temps » (time-slices en anglais) correspondant en chaque point de mesure à l'intégration de l'énergie réfléchie durant un certain intervalle de temps ${ }^{2}$. Il s'agit donc de cartes d'indices de réflectivité, obtenues à une certaine profondeur et «d'une certaine épaisseur » (déterminée par la position choisie en temps sur la coupe et la tranche de temps choisie sur cette même coupe). La conversion temps-épaisseur est obtenue en supposant que la vitesse du milieu prospecté est constante. Par ailleurs, cette vitesse est estimée à partir d'un profil dit en point milieu commun (Common-Mid-Point ou CMP). De telles cartes sont davantage conformes au mode de représentation des données que l'on est habitué à utiliser avec d'autres méthodes géophysiques ; elles permettent, par ailleurs, d'obtenir de façon quasiment automatique une représentation tridimensionnelle du sous-sol, beaucoup plus parlante que les réponses en coupe verticale du terrain - comme celle de la figure 3 - qui n'offrent, le plus souvent, que l'illusion d'une facilité de lecture et d'interprétation.

\section{Méthode électrostatique}

La méthode électrostatique est une généralisation de la méthode électrique aux milieux diélectriques ${ }^{3}$. Grâce aux fréquences utilisées (de l'ordre de $30 \mathrm{kHz}$ ), le contact galvanique des électrodes de la méthode électrique peut être remplacé par un effet de type capacitif sans que l'impédance vue par le circuit d'injection ne soit trop forte. Les pôles du dispositif sont des pièces métalliques isolées placées dans l'air et que l'on peut poser sur la surface du sol. Le procédé permet donc de réaliser des mesures de résistivité apparente indépendamment du type de surface, qu'elle soit conductrice (prairie) ou infiniment résistante (bitume). La mise en œuvre des mesures et l'interprétation des données sont en tout point similaires à ce qui est effectué avec la méthode électrique. En effet, la mesure est réalisée au moyen d'un quadripôle : deux pôles A et B servant à l'injection du courant et deux autres pôles $M$ et $N$ permettant d'effectuer la mesure de différence de potentiel. À courant constant, cette différence de potentiel $\Delta \mathrm{V}$ est proportionnelle à la résistivité apparente $\rho_{\mathrm{a}}$ du volume pris en compte dans la mesure :

$$
\rho_{\mathrm{a}}=2 \pi \frac{\Delta \mathrm{V}}{\mathrm{I}} \frac{1}{\frac{1}{\mathrm{AM}}-\frac{1}{\mathrm{AN}}-\frac{1}{\mathrm{BM}}+\frac{1}{\mathrm{BN}}}
$$

2. Cf. C. CAMI:RI.YNCK et al., 1994.

3. Cf: А. ТАвваgh et al., 1993. 
où I est l'intensité du courant injecté, $\mathrm{AM}, \mathrm{AN}, \mathrm{BM}$, et $\mathrm{BN}$ sont les distances entre les quatre pôles du dispositif.

La géométrie du dispositif est a priori quelconque. Cependant cette géométrie est souvent fixée par rapport à différents critères techniques et méthodologiques. Ainsi, la forme carrée offre un bon rapport «longueur du côté a (ou encombrement) / profondeur d'investigation », et sa réponse est relativement isotrope. Enfin, cette géométrie est bien adaptée aux dispositifs tractés permettant des mesures en continu ( $C f$. fig. lc et $4 a$ ). En revanche, l'exploration de profondeurs plus importantes (en général supérieures à $3 \mathrm{~m}$ ) est obtenue au moyen de dispositifs en ligne ( $C f$. fig. $1 \mathrm{~b}$ et 4b) qui se rapprochent plus facilement des obstacles ou se faufilent mieux dans les rues et les espaces restreints du milieu urbain.

Le volume concerné par la mesure dépend de la taille du dispositif ; ainsi avec un petit gabarit (carré de $50 \mathrm{~cm}$ de côté par exemple), il sera réduit et la profondeur d'investigation sera légèrement inférieure à $50 \mathrm{~cm}$. Il est donc nécessaire d'augmenter sa taille pour détecter des informations

Figure 4. Schémas de dispositifs électrostatiques de mesure de la résistivité apparente avec I et $V$ représentant respectivement des électrodes d'intensité et de potentiel

a. Vue en plan du multipôle électrostatique urbain (voir fig. Ic)

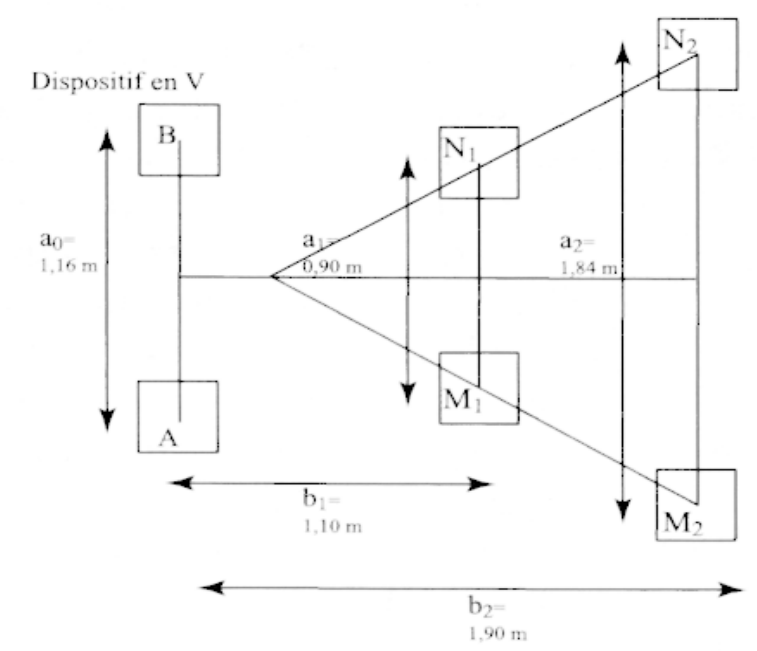

b. Configuration des électrodes en ligne de type Wenner pour le sondage électrostatique (voir fig. Ib)

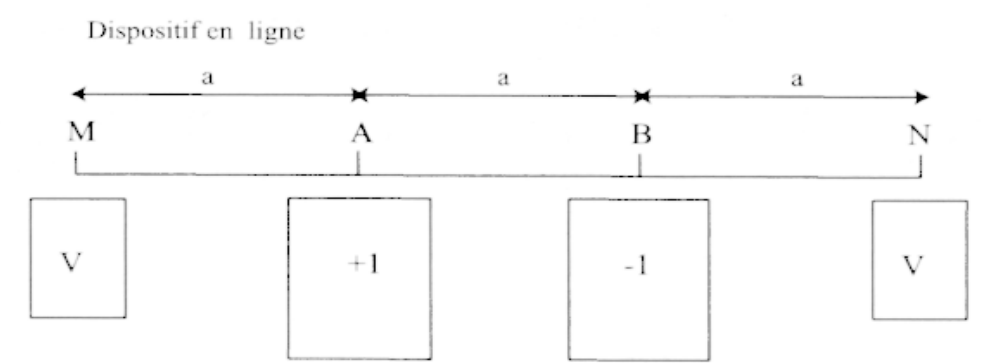


plus profondes. Le déplacement du système selon un maillage régulier permet ainsi de mettre en évidence les variations latérales de la résistivité électrique sur une largeur de terrain déterminée par la taille choisie. La réalisation de mesures avec plusicurs tailles de dispositifs (sondages) permet ensuite d'établir les variations verticales de la résistivité. Les multipôles développés ces dernières années $(C f$. fig. 1c) conjuguent ces deux informations en autorisant des mesures le long de profils avec plusieurs profondeurs simultanées ${ }^{4}$. Pour ces systèmes de mesure en continu, le positionnement est assuré par des appareils de type odomètre ou radar à effet Doppler. Des dispositifs modulaires ont été développés pour répondre aux différents contextes susceptibles d'être rencontrés en milieu urbain jusqu'à des profondeurs de trois mètres. Leur utilisation est en général couplée à des systèmes de pôles indépendants ( $C f$. fig. 1b) permettant la réalisation de sondages sur de plus grandes profondeurs pour pouvoir situer les structures dans leur environnement souterrain.

Ces deux méthodes permettent d'acquérir une description du sol en trois dimensions, qui est fort utile compte tenu des multiples remaniements subis par celui-ci et de la superposition de structures de nature et de forme très diverses. Elles mettent en évidence les contrastes à partir de deux paramètres physiques différents (permittivité électrique pour le radar et résistivité électrique pour la méthode électrostatique), dont les variations sont principalement dues aux inégalités dans la teneur en eau du milieu et à son contenu en argile. La première méthode est une méthode ponctuelle qui permet de détecter et séparer des objets de petite taille jusqu'aux profondeurs qui nous intéressent (environ $5 \mathrm{~m}$ ), avec une limitation en cas de milieu argileux; dans ce dernier cas, la méthode radar-sol est rendue inefficace par la forte atténuation de l'onde électromagnétique. La seconde méthode est mieux appropriée à la description des variations globales. Comme nous l'avons vu précédemment, sa résolution spatiale diminue avec la profondeur. Par ailleurs, la profondeur d'investigation maximale dépend de l'écartement des pôles, écartement qui est en général contraint par l'exiguïté de la surface prospectée. Néanmoins, et contrairement au radar, la méthode électrostatique peut être utilisée pour prospecter des milieux tant conducteur que résistant.

\section{Problématiques}

Le sol urbain est l'objet d'enjeux permanents. Il a une histoire, et son futur peut être l'objet d'âpres conflits. L'approche pluridisciplinaire est

4. Cf. C. PANissoid et al., 1998a et b. 
nécessaire à la compréhension de sa structure et de tous les phénomènes qui y sont liés (circulation d'eau, fuite des polluants, contraintes sur les fondations de bâtiments...). La reconnaissance de cette structure est une première étape qui doit permettre aux différents chercheurs d'évaluer le patrimoine présent et de l'insérer dans des problématiques de recherche. Celles-ci peuvent, a priori, être très larges, comme l'étude des modes d'urbanisation ou de l'évolution de l'habitat. Les fouilles réalisées en milicu urbain posent deux problèmes majeurs vis-à-vis de ces problématiques:

- elles correspondent à des surfaces souvent très limitées ;

- leur emplacement n'est pas maîtrisable dans la mesure où il est souvent (toujours ?...) dicté par la réalisation d'aménagements modernes. Compte tenu de la complexité du contexte, les campagnes de reconnaissance doivent donc être envisagées selon une stratégie bien définie et sur des objectifs précis.

La stratégie doit s'adapter aux situations et compléter les informations résultant d'éventuelles campagnes de fouilles antérieures. Parmi les objectifs, l'étude des vestiges des bâtis anciens sous les édifices actucls (cathédrales, simples églises ou autres) tient aujourd'hui une place importante et correspond à plusieurs programmes de recherche nationaux et européens. En se limitant aux travaux les plus récents, les principaux édifices auscultés par notre équipe à ce jour sont les suivants : cathédrales de Beauvais, de Chartres ', de Gérone et de Varsovie, églises de La Charité et Saint-Étienne de Nevers, abbaye Saint-Germain et cathédrale SaintÉtienne d'Auxerre... La détection de structures à l'intérieur de murs d'édifices (cathédrale de Reims, châteaux de Vincennes et Versailles) ou l'examen de problèmes liés à des modes de sédimentation (terres noires des occupations médiévales) ont également constitué des sujets de recherche. La reconnaissance des grands traits de la structure urbaine (réseaux de voies anciennes, fortifications, etc.), comme pour l'Heptastade d'Alexandrie ', les villes de Saint-Romain-en-Gal, Chartres, Saint-Denis ou La Rochelle, constitue cependant le premier objectif que l'on peut assigner à la prospection géophysique en milieu urbain.

\section{Exemples d'application}

\section{Prospection de la cathédrale de Gérone (Espagne)}

Cet exemple correspond à une prospection couplée électrostatique et radar qui a été menée en collaboration avec l'équipe d'Albert Casas

5. (\% M. DNBAS et al. 1993.

6. Cf. A. HI:SSI: et al., 1998. 
(Université de Barcelone) et dans le cadre du programme AIP Picasso ?. L'intérêt pour ce type d'édifices majeurs est très important dans la mesure où il s'agit en général de sites occupés depuis une période très ancienne. La cathédrale de Gérone n'échappe pas à cette règle. Il s'agit en effet d'une construction gothique (1350), remplaçant une construction romane du $\mathrm{XI}^{\mathrm{C}}$ siècle, qui devait elle-même se superposer à des vestiges romains. La cathédrale actuelle est caractérisée par sa nef dont la largeur de $23 \mathrm{~m}$ en fait la nef la plus large du monde. Les questions posées sur ce site correspondaient donc à la détection des vestiges de la cathédrale romane, et éventuellement à celle de vestiges antérieurs. La prospection s'intégrait également à une stratégie de fouilles dans la mesure où elle devait permettre de déterminer la zone la plus intéressante pour réaliser une campagne programmée.

La prospection électrostatique a été réalisée au moyen d'un quadripôle électrostatique carré de côté $\mathrm{a}=1,2 \mathrm{~m}$ (ESR-66, Cnrs). Le prototype utilisé pour cette expérience est tracté manuellement et permet la mesure en continu de la résistivité apparente ( $C f$. fig. lc pour la version opérationnelle de l'appareil). L'échantillonnage était de $5 \mathrm{~cm}$ le long des profils avec un espacement de $1 \mathrm{~m}$ entre chacun d'eux. Après ré-interpolation, la carte de résistivité apparente a été tracée selon un maillage carré régulier de $0,25 \mathrm{~m}$.

Les données radar ont été acquises avec un appareil Pulse Ekko 1000 (Sensors and Software, Toronto) doté d'une antenne de fréquence $450 \mathrm{MHz}$. Les mesures ont été effectuées manuellement avec un échantillonnage de $10 \mathrm{~cm}$ le long des profils et une distance de $1 \mathrm{~m}$ entre chacun d'eux. Pour la carte de résistivité apparente ( $C f$. fig. 5), les structures résistantes sont en foncé et les structures conductrices en clair. Sur la carte radar ( $C f$. fig. 6), les éléments les plus réfléchissants sont foncés, et, inversement, les moins réfléchissants, clairs. La carte radar centrée sur $14 \mathrm{~ns}$ (temps double), soit une profondeur d'environ $0,9 \mathrm{~m}$ (avec une vitesse de $0,125 \mathrm{~m} / \mathrm{ns}$ déduite des profils CMP), peut être comparée à la carte électrostatique dont la profondeur d'investigation est du même ordre de grandeur.

On distingue sur ces cartes la présence d'un certain nombre de petites anomalies situées sur les côtés nord et sud de la nef, ainsi qu'à la limite est de la prospection à l'entrée du chœur : elles apparaissent ponctuclles sur la carte électrostatique. De plus, on distingue deux rangées d'anomalies situées en bordure des cartes radar : elles correspondent à la présence de tombes dont les dalles sont repérées en surface. La comparaison des deux types de données met en évidence la différence de résolution latérale des

7. Cf. M. DABAS \& C. CAMLRLYNCK, 2000. 
Figure 5. Prospection électrostatique (quadripôle carré $a=1,2 \mathrm{~m}$ ) de la cathédrale de Gérone

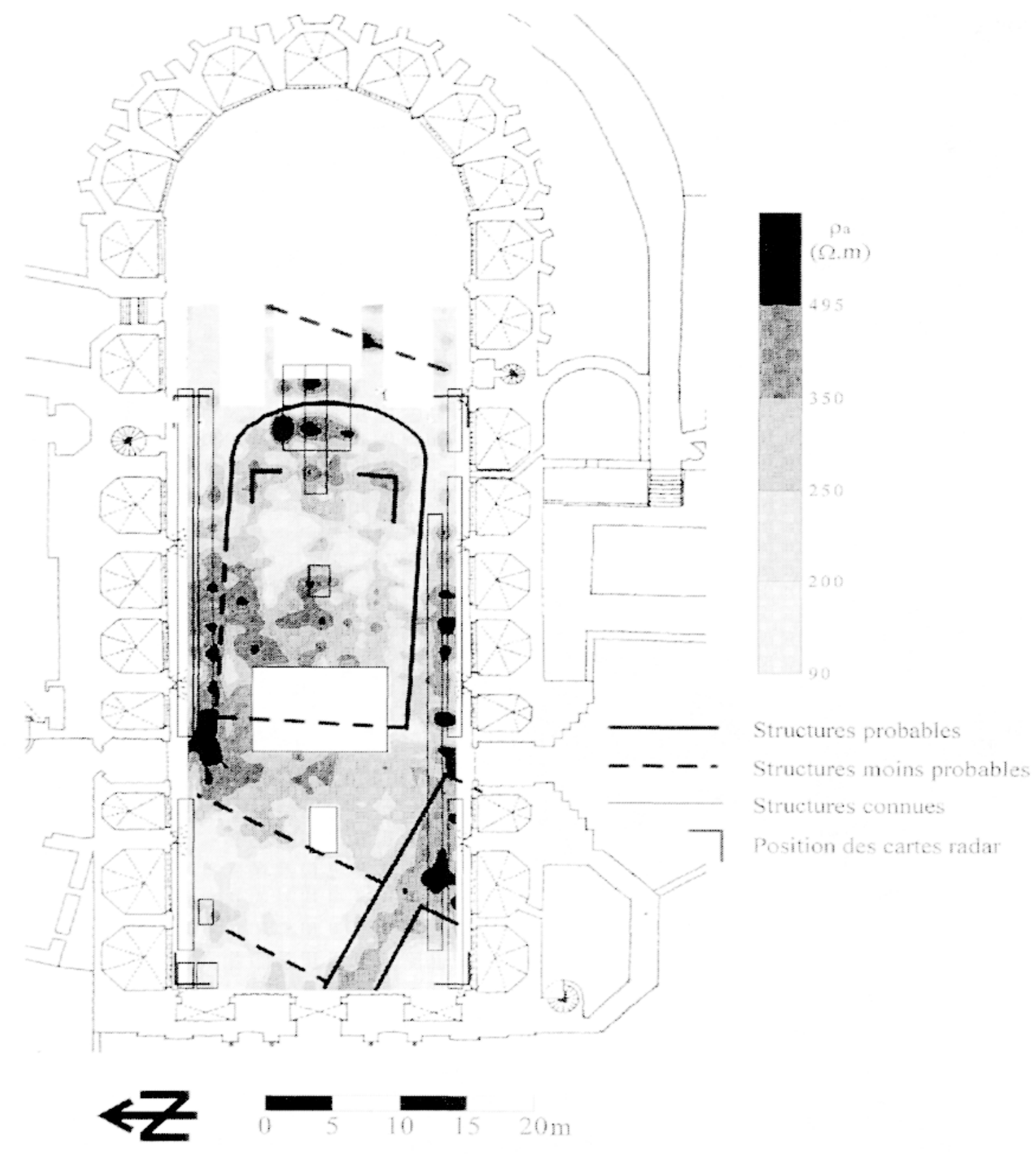

On observe de petites anomalies résistantes (en noir) sur les bords de la nef et à l'entrée du chœur qui correspondent à la présence de tombes. Une structure dont l'orientation differe d'environ $5^{\circ}$ d'avec la cathédrale actuelle est marquée à l'ouest par un fossé conducteur. On peut également distinguer une anomalie résistante sud-est/nord-ouest d'une largeur supérieure à deux mètres dans l'angle sud-ouest de la cathédrale, anomalie qui correspond aux soubassements d'une tour mise en évidence par les fouilles qui ont suivi. 
Histoire \& Mesure, 1999, XIV-3/4

Figure 6. Prospection radar de la cathédrale de Gérone
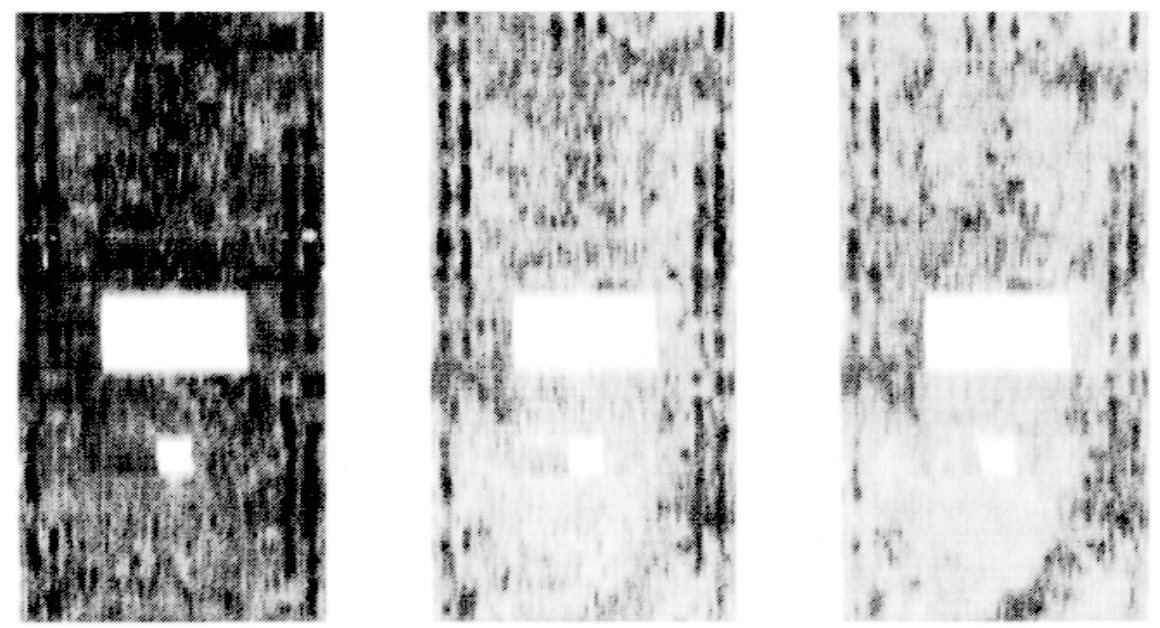

$4-8 n s$
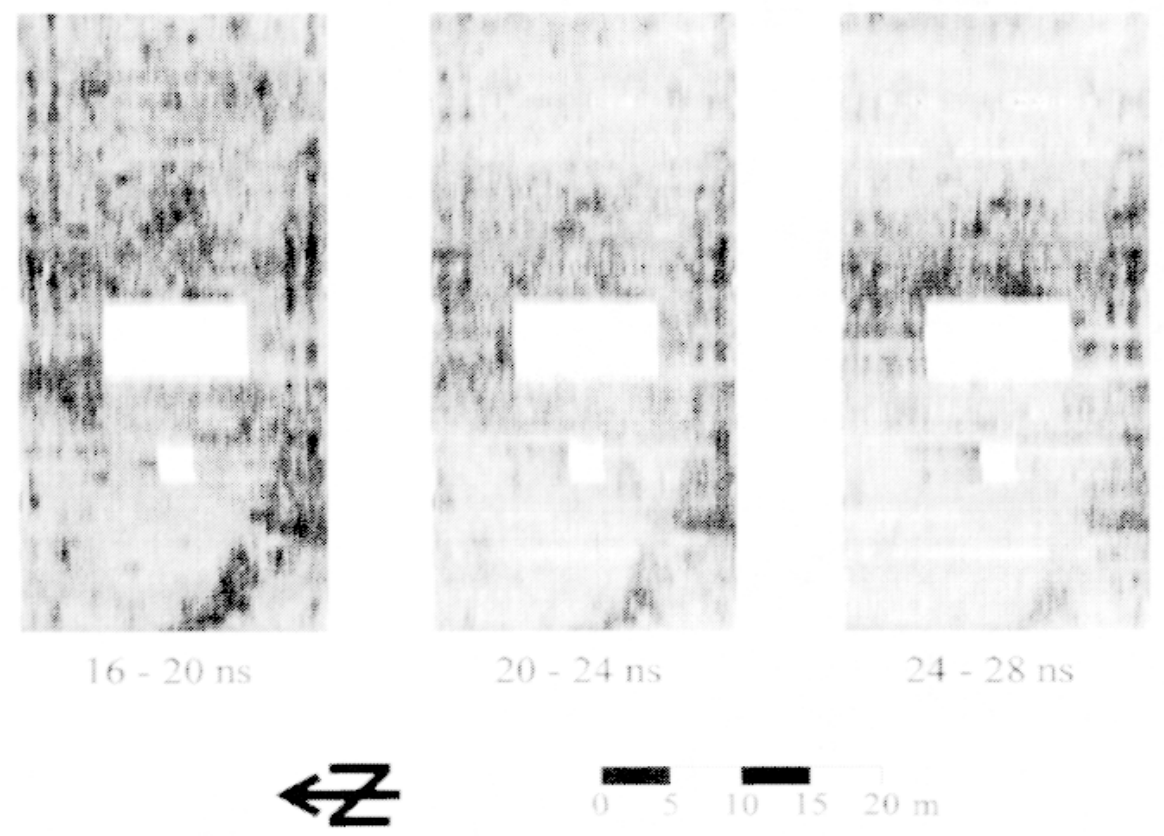

Chaque image représente une «tranche de temps » correspondant à l'intervalle indiqué en nano-secondes de la plus superficielle $(4-8 \mathrm{~ns}$ soit $0,25-0,5 \mathrm{~m})$ à la plus profonde (24-28 ns soit 1,5-1,75 m). L'image radar permet d'individualiser les deux rangées de tombes présentes de part et d'autre de la nef. Une structure fortement réfléchissante (en noir) est présente dans l'angle sud-ouest de la cathédrale.

deux méthodes. Le pouvoir de séparation plus grand du radar permet de distinguer les deux rangées de tombe, voire d'individualiser les différentes tombes dans ces rangées, ainsi que trois autres sépultures à l'entrée du chœur. Les données radar apportent par ailleurs une information sur la 
position et l'extension verticale de ces tombes. En effet, il s'agit d'anomalies superficielles qui apparaissent sur la carte la moins profonde (6 ns soit $0,4 \mathrm{~m}$ ) et disparaissent vers $18 \mathrm{~ns}$ (soit $1,15 \mathrm{~m}$ ). La forte résistivité (parfois supérieure à $500 \Omega . \mathrm{m}$ ) ainsi que le fort indice de réflectivité, observés pour ces anomalies, pourraient être corrélés à la présence de vides.

La lecture globale de ces cartes et en particulier de la carte électrostatique fait apparaître une structure dont l'orientation diffère d'environ $5^{\circ}$ d'avec la cathédrale actuelle. Elle est marquée à l'Ouest par un fossé conducteur curviligne, interprété selon les structures continues noires, qui pourraient correspondre aux fondations d'un édifice plus ancien (cathédrale romane ?). Enfin, l'angle sud-ouest de la prospection montre également une très grande cohérence des anomalies entre les deux types de méthode. On distingue une anomalie résistante et réfléchissante orientée sud-est/nord-ouest avec une limite quasiment à angle droit vers le sud-ouest. Ces anomalies, visibles jusqu'à 24 ns sur les cartes radar, pourraient correspondre à des structures construites. C'est dans cette zone que les archéologues ont finalement choisi de réaliser un sondage. Ces fouilles ont permis de prouver l'existence d'un édifice roman dont l'orientation diffère d'environ $3^{\circ}$ par rapport à la construction actuelle. S'ils n'ont pu vérifier les hypothèses émises quant à l'anomalie curviligne conductrice située devant le chœur, les fouilles ont en revanche permis de découvrir une tour qui était à l'origine de l'anomalie résistante située sur la face sud de la cathédrale.

\section{Prospection de la place de Verdun à La Rochelle (France)}

Cette prospection a fait partie d'une étude préliminaire au projet de construction d'un parking souterrain sous cette place située dans la vieille ville de La Rochelle. Des sondages archéologiques effectués à l'est de la zone prospectée avaient mis au jour des vestiges à une profondeur de l'ordre de $60 \mathrm{~cm}$. D'après les plans des fortifications, la place située à l'intérieur du rempart correspond à l'emplacement d'un château médiéval. L'objectif de la prospection était donc de détecter la présence éventuelle de structures résistantes qui correspondraient à des éléments de fondations et/ou de murs de cet ensemble fortifié. Il s'agit là de la première prospection électrostatique effectuée en milieu urbain sur une grande surface et avec un système multipôle. La prospection a en effet été réalisée à deux profondeurs d'investigation correspondant à $a_{1}=0,7 \mathrm{~m}$ et $\mathrm{a}_{2}=1,3 \mathrm{~m}$ (ESR-66, Cnrs). Avec ce multipôle électrostatique, la mesure de la résistivité apparente se fait en continu simultanément sur les deux voies de l'instrument. Le déplacement au sol est contrôlé par un odomètre et les profils sont visualisés en temps réel sur l'écran de l'ordinateur portable où sont stockées les données. La distance, séparant deux profils, a été fixée à 
$1 \mathrm{~m}$ pour toutes les zones et le pas d'échantillonnage le long des profils est de $10 \mathrm{~cm}$. L'ensemble des surfaces prospectées avec le multipôle électrostatique correspond environ à 0,5 ha.

Les résultats de cette prospection sont représentés sous la forme de cartes en niveaux de gris (résistant en foncé et conducteur en clair); deux cartes de résistivité apparente par zone correspondant à deux profondeurs d'investigation différentes ont été ainsi obtenues pour la prospection électrostatique. Ces cartes ont ensuite été intégrées à la carte du site $(C f$. fig. 7a et 7b) qui fournit un certain nombre d'indications notamment sur les différents concessionnaires. Ceci nous permet d'une part de lier la présence d'anomalies à des «objets » connus du sous-sol (par exemple, une conduite d'eau), et d'autre part de comparer les orientations des principales anomalies dans l'ensemble des zones prospectées. Pour l'interprétation de ces données, il convient de considérer deux zones de l'espace prospecté :

- la place de Verdun proprement dite : elle est couverte par un ensemble de trois cartes presque contiguës, à l'est de l'espace étudié. Les fouilles effectuées en 1994 et 1995 le long du côté nord de la place, complétées par une série de sondages en mars 1996, avaient mis au jour une tour supposée appartenir à la courtine nord du château, ainsi que des vestiges de cette même courtine ( $C f$. fig. 8 ). Les mesures effectuées au nord de la place (petite carte) mettent en évidence des anomalies résistantes qui semblent se raccorder aux alignements clairement identifiés à l'ouest de la place, sur les deux cartes plus au sud. Néanmoins, les murs de cette courtine nord ne peuvent être reconnus. En revanche, les grands alignements résistants situés à l'Ouest pourraient correspondre à des murs de la courtine. La partie est de cette zone fait apparaître un ensemble d'anomalies résistantes très organisé. La présence d'une anomalie à angle droit ainsi que la succession d'anomalies est-ouest selon un espacement de l'ordre de $6 \mathrm{~m}$ fait penser qu'il puisse d'agir de murs de soubassement d'un ou de plusieurs édifices différents. La surface de cet ensemble couvre environ $30 \mathrm{~m}$ sur $30 \mathrm{~m}$. L'anomalie circulaire résistante située en bordure sud de la place ne semble pas s'intégrer à l'organisation d'ensemble des autres anomalies; par ailleurs, sa signature est relativement différente : elle est très fortement marquée sur les deux profondeurs d'investigation. Il pourrait s'agir d'une structure plus récente. L'hypothèse des fondations d'un kiosque à musique occupant le centre de la place entre les milieux des $\mathrm{XIX}^{\mathrm{c}}$ et $\mathrm{XX}^{\mathrm{c}}$ siècles $\mathrm{n}^{\prime}$ 'st pas à écarter.

- la zone à l'ouest de la place de Verdun : elle a été principalement couverte par deux cartes dont l'allongement est orienté nord-sud. L'absence de continuité des mesures entre les deux zones est due à la présence des places de stationnement des bus, où seuls quelques profils (non représentés ici) ont pu être réalisés. Les alignements quasi nord-sud de 
Figure 7. Prospection électrostatique (multipôle) de la place de Verdun à La Rochelle

a. Petit quadripôle $a=0,7 \mathrm{~m}$

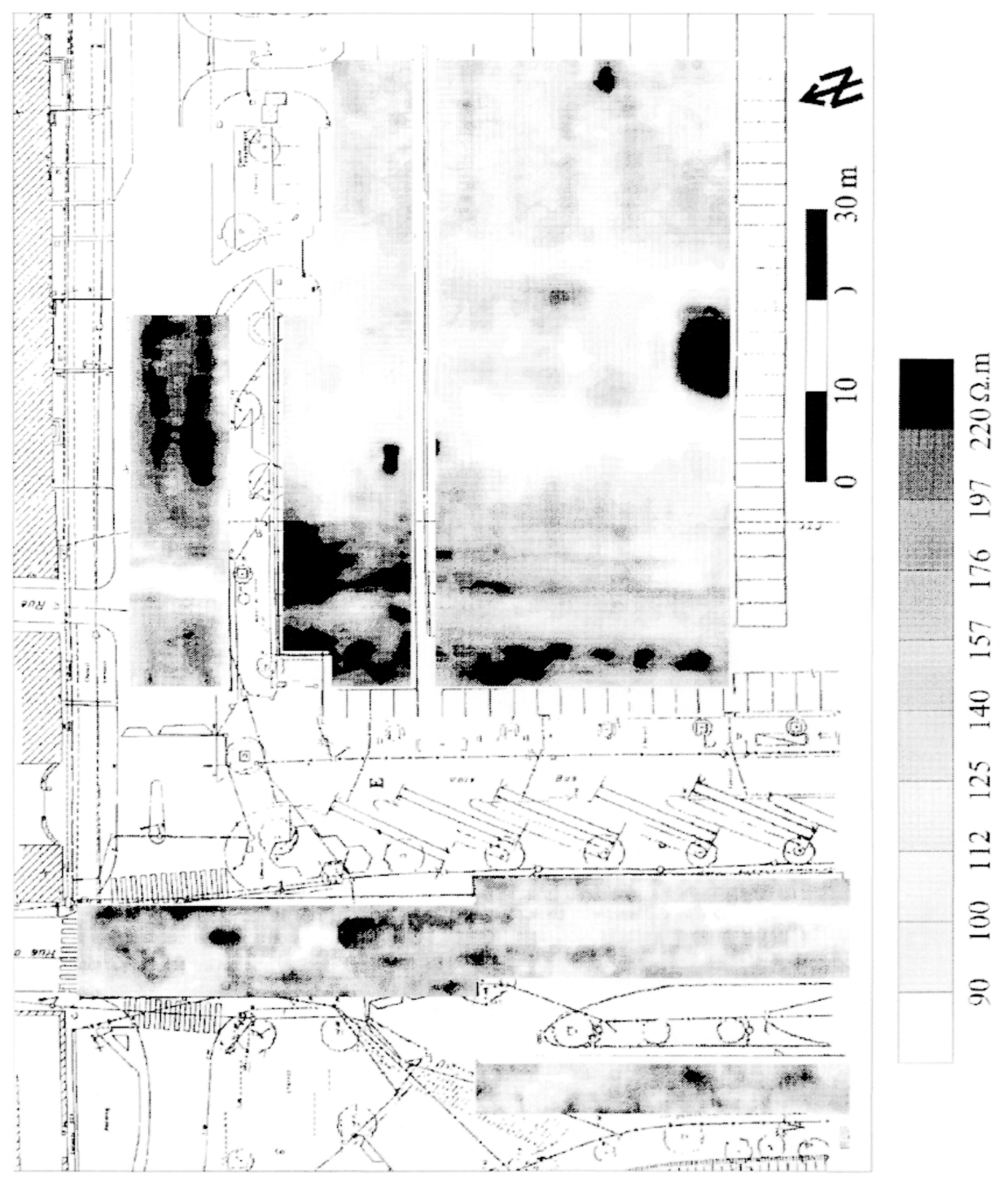


Histoire \& Mesure, 1999, XIV-3/4

b. Grand quadripôle $a=1,3 \mathrm{~m}$

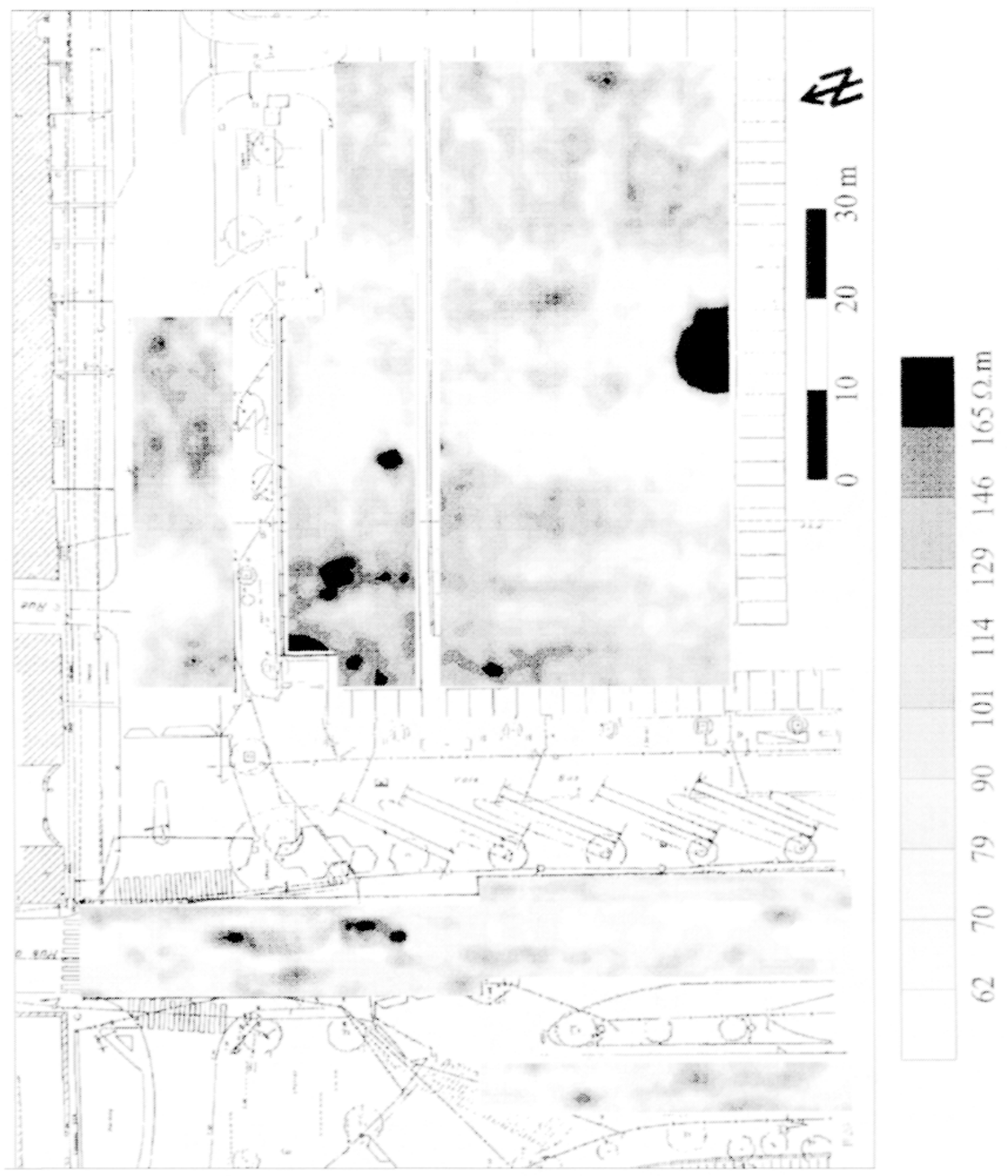

Ces cartes mettent en évidence un certain nombre d'alignements résistants sur la place, orientés nord-est/sud-ouest et est/ouest qui pourraient correspondre aux vestiges des murs de courtines ou de soubassements d'édifices. On note par ailleurs la présence d'une anomalie résistante circulaire identifiée comme correspondant aux vestiges d'un kiosque à musique. À l'ouest de la place, on observe d'autres anomalies résistantes dont l'orientation diffère avec celles observées sur la place. 
cette prospection ne semblent pas appartenir au même ensemble de structures que ceux qui sont à l'origine des anomalies observées plus à l'est, sur la place car leur orientation diffère sensiblement. Un plan du $\mathrm{XVIII}{ }^{c}$ siècle ( $C f$. fig. 9) mentionne la présence à cet endroit de murailles de soutènement de la « Courtine des Capucins » et de petits murets dans le "Bois d'Amourette ». Le positionnement de ces structures par rapport aux rues situées à l'angle nord-ouest et aux bâtiments bordant cette partie de la place permet d'envisager un lien entre les alignements et la « Courtine des Capucins ». Le tracé des rues ne semble pas avoir été modifié dans cette partie de la vieille ville. Sur ce plan du XVIII ${ }^{c}$ siècle, la place porte le nom de place du Château. Il est probable que, depuis cette époque, elle n'a plus été occupée par des bâtiments, et les structures à l'origine des anomalies doivent être bien antérieures à ce plan.

La prospection électrostatique réalisée sur la place de Verdun à La Rochelle dans le cadre d'une intervention de « sauvetage » montre l'intérêt de l'application de cette méthode à la détection de structures archéologiques en milicu urbain. Outre le fait que l'intervention a été sollicitée et décidée dans un délai de l'ordre d'une semaine, sa mise en couvre a été très rapide et s'est adaptée facilement aux contraintes liées à la circulation automobile en milieu urbain. Deux personnes au maximum sont nécessaires à la réalisation d'une telle prospection. Aucun câble ne traverse les voies de passage dans la mesure où tout le système de mesure et d'acquisition est embarqué sur le dispositif.

Figure 8. Plan de La Rochelle daté de 1621 (échelle inconnue)

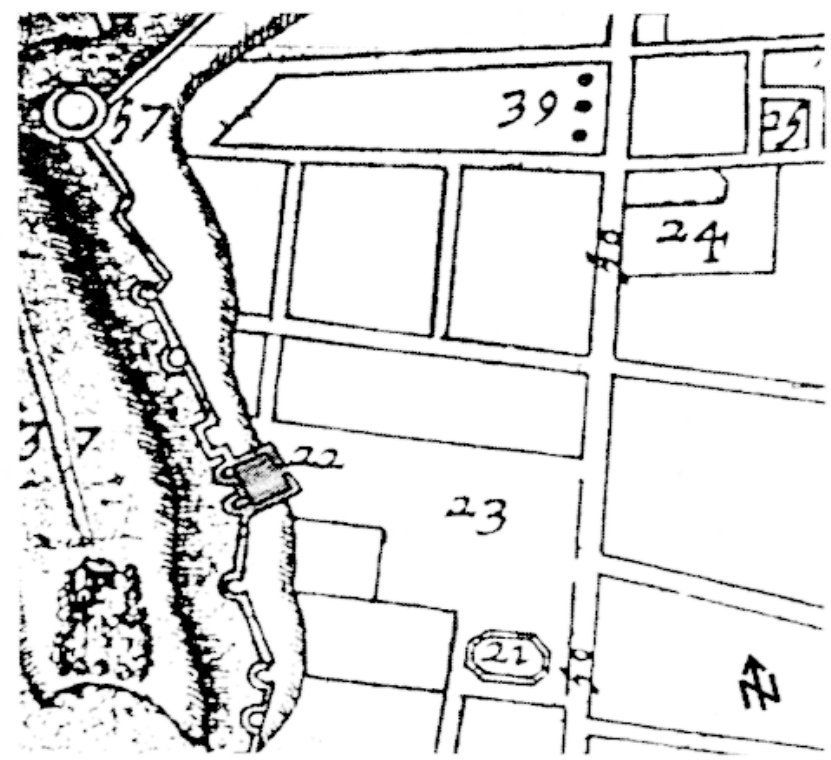


Figure 9. Plan de La Rochelle daté de 1743 (échelle inconnue)

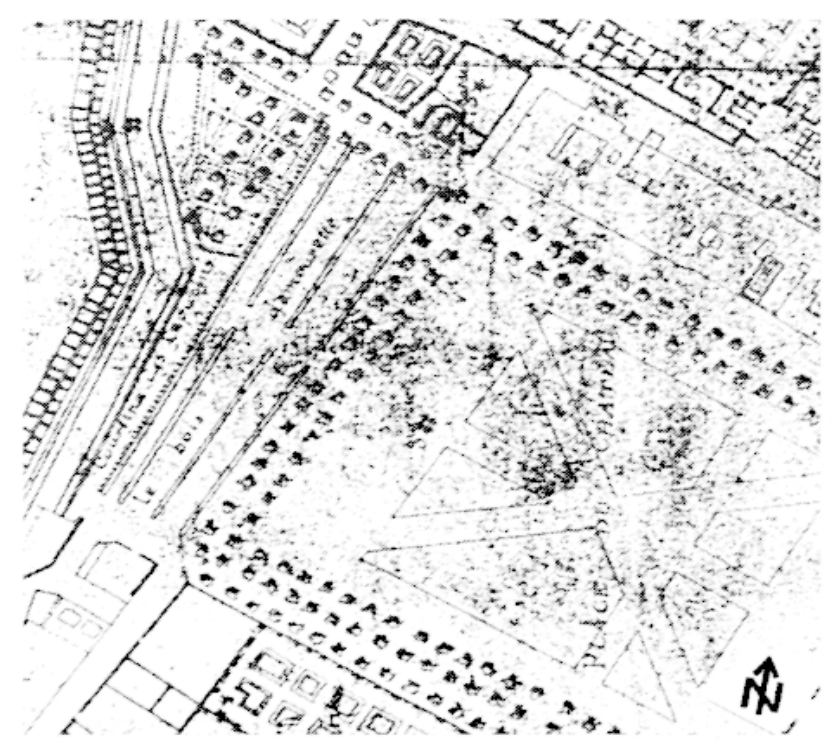

\section{Prospection des rues de l'isthme d'Alexandrie (Égypte)}

Le troisième exemple est celui de la recherche de l'Heptastade, chaussée antique longue de sept stades (environ $1155 \mathrm{~m}$ ) qui reliait la ville d'Alexandrie à l'île de Pharos. Cette étude s'intègre dans le programme d'archéologie urbaine et de fouilles sous-marines conduit par le Centre d'Études alexandrines sous la direction de J.-Y. Empereur. Une hypothèse émise au milieu du XIX $X^{\mathrm{c}}$ siècle par Mahmoud-Bey plaçait cet Heptastade au milieu de l'isthme actuellement existant, en discordance avec le réseau antique des rues ( $C f$. fig. 10). Cette discordance et un examen de l'organisation des rues ont conduit A. Hesse à effectuer une nouvelle séric de recherches pour préciser cette position ${ }^{8}$ et, si possible, la confirmer grâce à une campagne géophysique multi-méthodes. Cette reconnaissance a, tout d'abord, été basée sur une synthèse de la documentation historique (principalement les écrits de Strabon qui est un des rares auteurs à décrire l'Heptastade) et topographique (étude des différents cadastres). Un tel exemple illustre parfaitement l'ensemble des difficultés de mesure et d'interprétation liées à la reconnaissance géophysique des sols en milieu urbain. Ces difficultés sont d'autant plus grandes qu'il s'agit de suivre une structure « linéaire », l'Heptastade, qui pourrait se trouver enfouie à une profondeur de plusieurs mètres et dont la constitution nous est totalement inconnue.

8. Cf. A. HESSI: 1998. 
Figure 10. Situation de l'Heptastade selon l'hypothèse émise par A. Hesse, dans l'axe $H$ de la rue $R 9$, et selon la publication de $M$. Bey; dans l'axe $M$

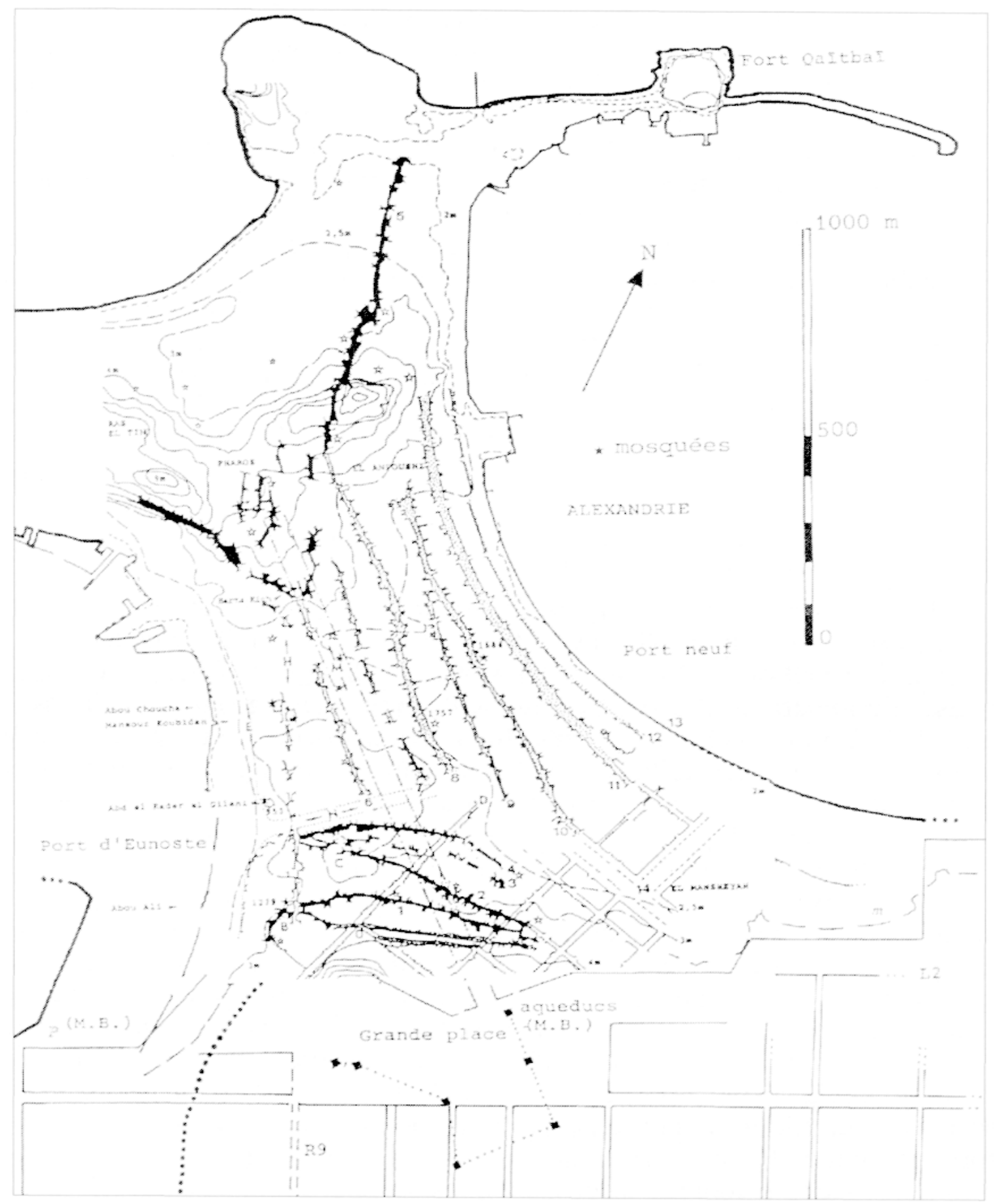

Une prospection multi-méthodes a donc été organisée dans un certain nombre de rues de l'isthme, posant clairement un problème d'échantillonnage. Les rues choisies, sur des distances d'environ $300 \mathrm{~m}$ en moyenne, 
recoupent perpendiculairement l'Heptastade conformément à l'hypothèse émise par A. Hesse. L'eau salée présente à une profondeur inférieure à $5 \mathrm{~m}$ constituait également une difficulté majeure. Les méthodes employées ont été la méthode sismique réfraction (Mark6, ABEM), le radar-sol (Pulse Ekko 100, 50M Hz et $100 \mathrm{MHz}$ ), la méthode Slingram (EM31, Geonics) et la méthode électrostatique (ESR3, Cnrs). Les données sismiques n'ont pu apporter d'informations satisfaisantes, la source (frappe au marteau sur une plaque métallique posée sur le sol) s'étant avérée trop faible et le bruit environnant trop pénalisant. Les données Slingram (conductivité apparente), bien que fortement bruitées par la présence d'objets métalliques et de lignes électriques, ont montré, après un important filtrage, des résultats très cohérents avec les mesures de résistivité apparente obtenues avec la méthode électrostatique. Enfin, les coupes radar ont permis de mettre en évidence un certain nombre de frontières et de structures réfléchissantes. L'ensemble des données ${ }^{9}$ suggère que l'Heptastade ne constitue pas un marqueur au sens géophysique du terme. Aucun des différents types de données ne permet, en effet, de suivre d'une rue à l'autre une anomalie étroite et rectiligne que l'on pourrait associer à une structure que, peut-être à tort d'ailleurs, on imagine construite. Néanmoins, il existe un noyau résistant, avec des limites relativement précises, qui coïncide avec le tracé de la nouvelle hypothèse.

La prospection électrostatique a consisté en la réalisation de profils Wenner ( $C f$. fig. $1 \mathrm{~b}$ et $4 \mathrm{~b}$ ) avec trois écartements différents (a $=3 \mathrm{~m}$, $a=6 \mathrm{~m}$ et $\mathrm{a}=9 \mathrm{~m}$ ), qui révèlent les variations latérales et verticales de la résistivité apparente. Le petit écartement, avec une profondeur d'investigation de l'ordre de $2 \mathrm{~m}$, a essentiellement permis de mettre en évidence la forte hétérogénéité de la couche superficielle (fortement remaniée). Les deux autres écartements correspondaient davantage à l'intervalle de profondeur dans lequel devait se situer la structure recherchée. Les différents profils ont fait apparaître un niveau moyen de résistivité faible, inférieur à $10 \Omega . \mathrm{m}(C f$. fig. 11$)$, qui correspond à des niveaux sableux saturés d'eau salée. Les sondages effectués dans d'autres rues confirment la présence de ces niveaux très conducteurs $(5 \Omega . \mathrm{m})$ sous une couche superficielle dont l'épaisseur varie entre un et deux mètres et dont la résistivité est de l'ordre de 30-40 $\Omega$.m ( $C f$. fig. 12). La carte électrostatique (Cf. fig. 13), obtenue par interpolation entre les profils effectués au moyen d'un dispositif de type Wenner a $=6 \mathrm{~m}$, révèle l'existence d'une anomalie résistante au milieu de la rue $\mathrm{E}$, qui correspond également à une anomalie topographique ( $C f$. fig. 14). D’autres zones résistantes (en comptant celle donnée par des sondages électrostatiques réalisés entre les rues $\mathrm{B}$ et $\mathrm{C}$ ) semblent constituer un alignement qui, bien qu'interrompu par des zones

9. Cf. A. Hissi et al., 1998. 
très conductrices (rues $\mathrm{B}$ et $\mathrm{D}$ ), est conforme au nouveau tracé proposé. On serait donc finalement tenté d'associer cet alignement nord-ouest/sud-est et les noyaux résistants à des traces « fossiles » de l'ensablement de l'isthme, ensablement qui aurait pu se faire de façon privilégiée autour d'îlots rocheux situés entre la côte et l'île de Pharos. Par ailleurs, Strabon ayant décrit l'Heptastade comme une sorte de pont comportant deux passages entre les ports est et ouest, il est très possible que les zones conductrices soient la marque de l'un ou l'autre de ces passages situés entre deux îlots rocheux. ${ }^{10}$

Figure 11. Profils de résistivité apparente obtenus dans la rue Abd el Moneim el Deba (D)
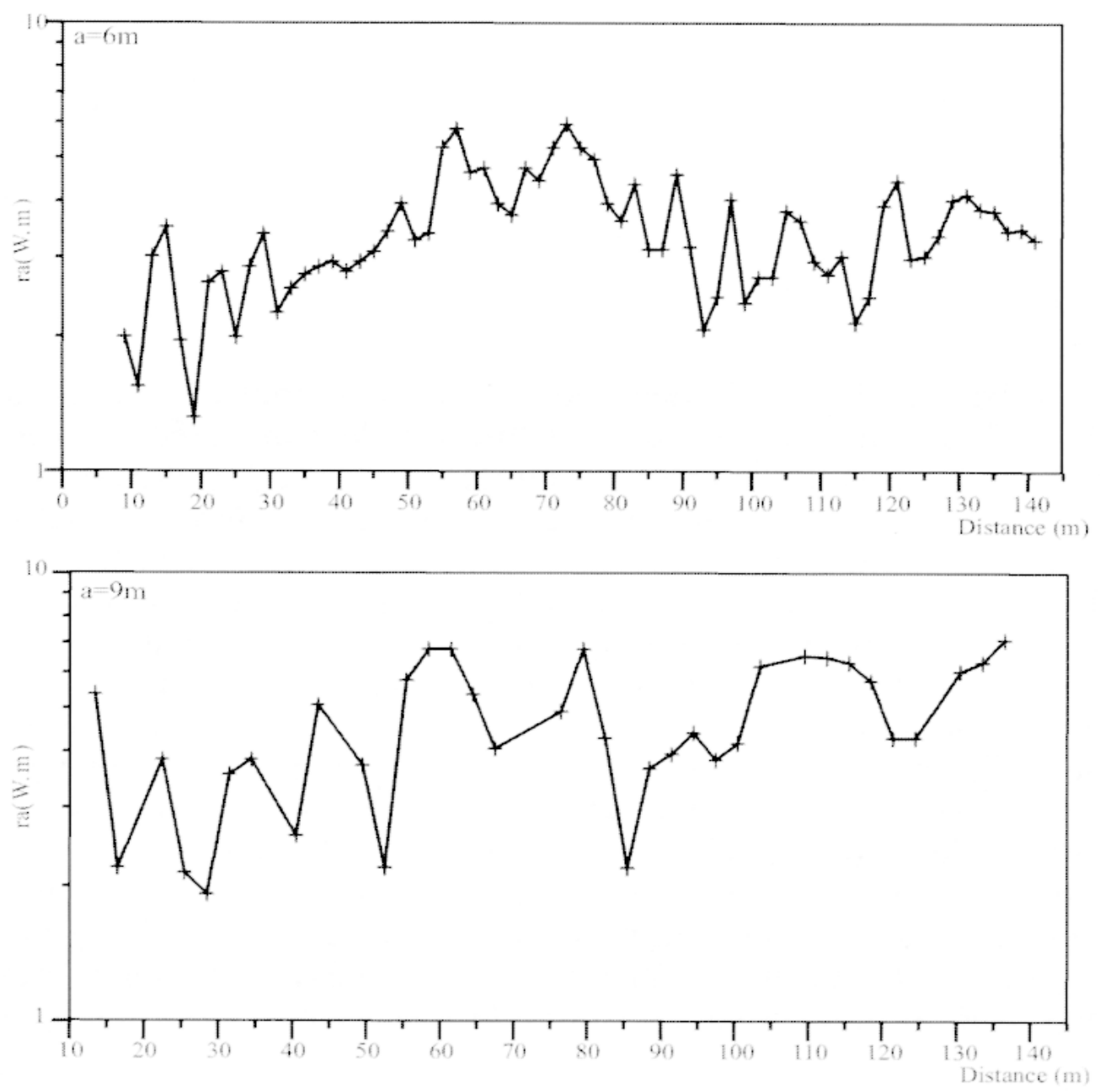

10. Cf. A. Hr:Ssi: 1998a. 
Figure 12. Sondage électrostatique (SI) réalisé dans la rue Kabou el Mallah

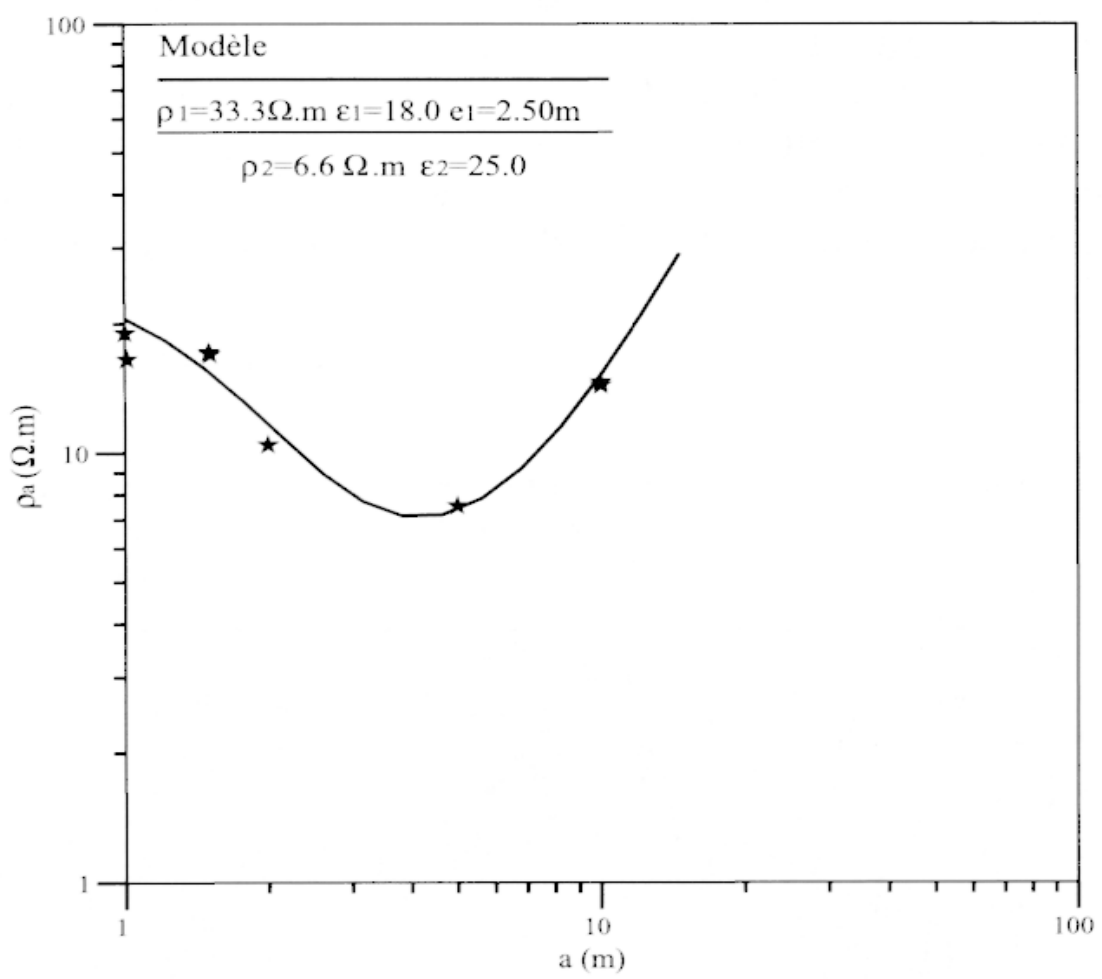

L'utilisation des méthodes géophysiques permet d'acquérir des informations dans un milieu où la réalisation de sondages destructifs et onéreux est impossible de façon systématique. Les difficultés propres aux milieux urbains (forte hétérogénéité sur plusieurs mètres, superposition des structures, interpénétration des interventions humaines) imposent la définition d'un protocole de mesure adapté et l'utilisation simultanée d'au moins deux méthodes. Les premiers exemples réalisés dans le cadre de différentes problématiques archéologiques ont montré que l'association des méthodes radar et électrostatique constitue une combinaison permettant de s'assurer de bons résultats dans une grande partie des situations rencontrées à ce jour. Avec le développement de l'utilisation de ces outils, leur meilleure connaissance, ainsi que leur adaptation technique aux problèmes des sols historiques urbains, le champ des problématiques auxquels ils peuvent être appliqués devrait s'élargir ces prochaines années. Enfin, il faut envisager de nouveaux développements méthodologiques tant au niveau des appareils de mesure que des traitements qui leur sont associés. 
Cédric Panissod \& Michel Dabas

Figure 13. Prospection électrostatique Wenner $a=6 m$ à Alexandrie

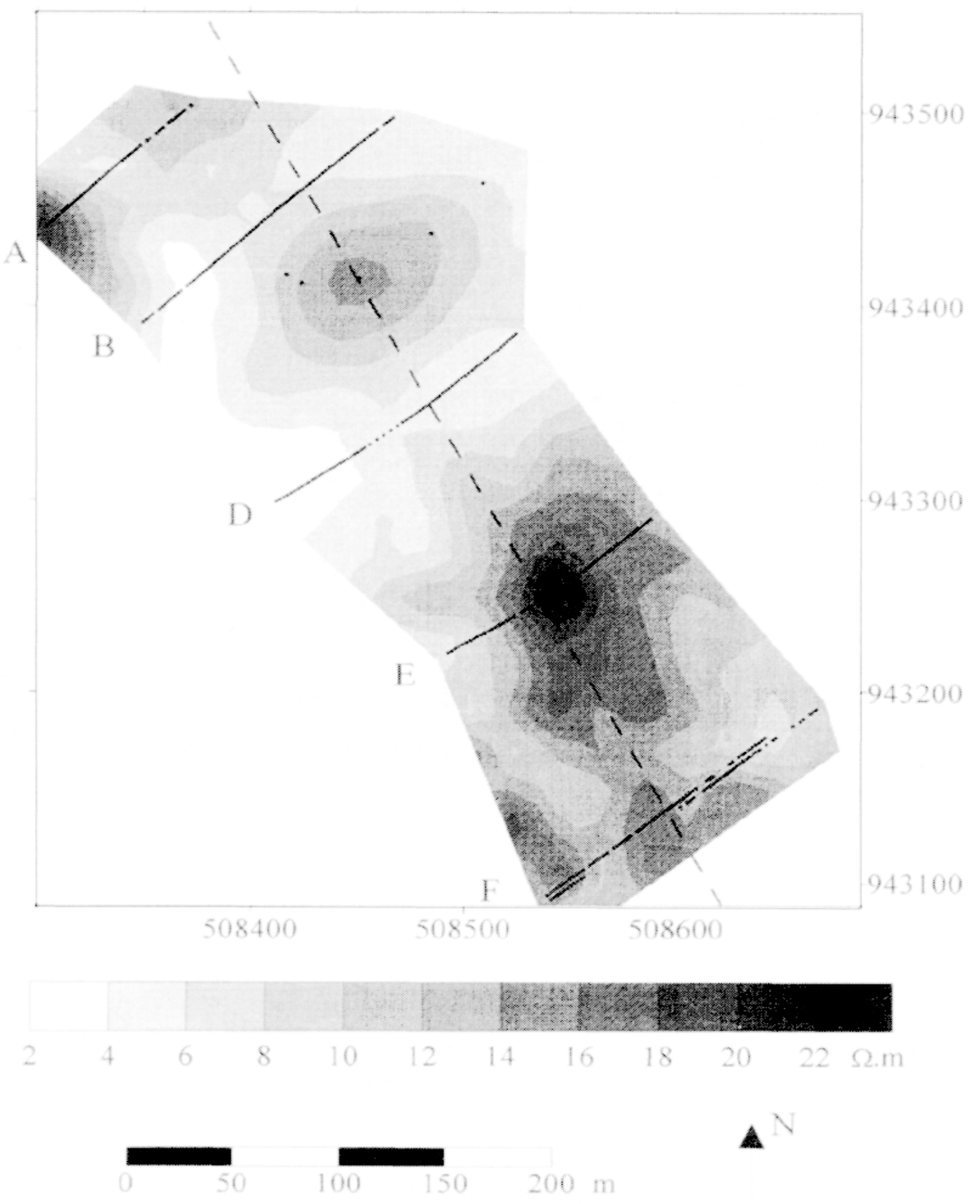

Les tracés en noir marquent les profils explorés dans les rues de l'isthme; les points noirs, les positions des sondages électrostatiques; la ligne en tirets, l'axe de la nouvelle hypothèse de tracé. On constate qu'un certain nombre d'anomalies de forte résistivité (en gris et noir) s'alignent bien sur l'axe proposé pour l'Heptastade et que les résistivités s'abaissent (en plus clair) de part et d'autre. 
Figure 14. Topographie de la zone prospectée à Alexandrie

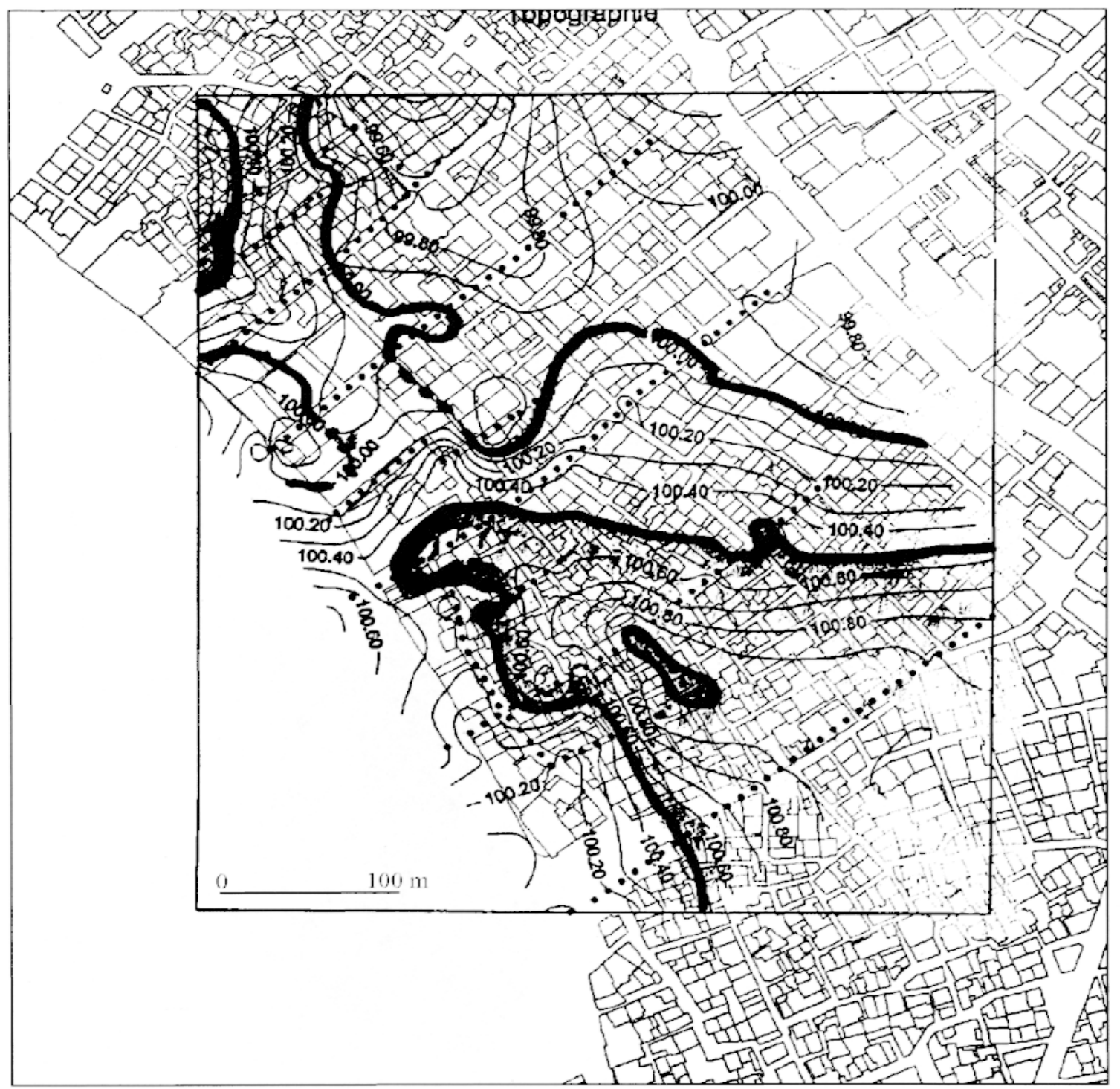

Cadastre de 1930 en fond de plan; situation des profils explorés dans les rues en pointillés; altitudes de la surface des chaussées en courbes de niveau ( 0 arbitraire, intervalle $0,1 \mathrm{~m}$ ). On observe, en se repérant par rapport aux profils, que les altitudes les plus élevées se trouvent au voisinage de l'Heptastade (en ligne de tirets) et qu'elles correspondent aux résistivités les plus fortes cartographiées sur la figure 13.

\section{BIBIIOGRAPHIE}

CAmIRI.ynck, Christian, Dabas, Michel \& PANissod, Cédric, "Comparison between GPR and four electromagnetic methods for stone features characterisation : an example », Archaeological Prospection, 1994, 1, pp. 5-17.

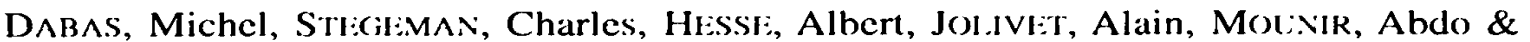
CASAS, Albert, "Prospection géophysique dans la cathédrale de Chartres », Bulletin de la Société Archéologique d'Eure-et-Loir, 1993, n 36, $1^{\text {er }}$ trim., pp. 5-25. 
DABAS, Michel \& CAMLRI.YNCK, Christian, "Simultaneous use of electrostatic quadrupole and GPR in an urban context : investigation of the basement of the cathedral of Girona (Catalunya-Spain) », Geophysics, 2000), 65, 2, pp. 1-12.

HI:SSE, Albert, "Arguments pour une nouvelle hypothèse de localisation de l'Heptastade d'Alexandrie ", Ëudes alexandrines, 1, J.-Y. Empereur, 1998, IFAO, Le Caire, pp. $21-33$.

Hrssi:, Albert, JOIJVI:T, Alain \& TABBAGiH, Alain, "New prospects in shallow depth electrical surveying for archacological and pedological applications », (jeophysics, 1986,51 , pp. 585-594.

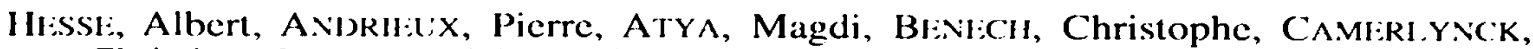
Christian, Dabas, Michel, Fíchant, Catherine, Jolivit, Alain, Ki:NT, Cécile,

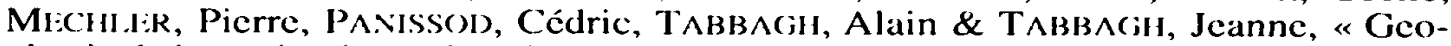
physical investigations for the location of the Heptastadium $»$, in Alexandria (Egypt), 4th meeting of E.E.G.S., Envirommental and engineering geophysics, Barcelona, expanded abstracts, sept. 4-17 1998, 1998, pp. 715-718.

Panissom, Cédric, Dabas, Michel, Hessi:, Albert, Jolivit, Alain, Tabbagh, Jeanne \& TABBAciH, Alain, "Recent developments in shallow depth electrical and electrostatic prospecting using mobile arrays » (Geophysics, 1998a, 63, 5, pp. 1542-1550.

PANissod, Cédric, DABAs, Michel, Fiorsch, Nicolas, Hessi:, Albert, Jolivit, Alain, TABBAciH, Alain \& TABBAGH, Jeanne, "Archacological prospecting using electric and electrostatic mobile arrays », Archaeological Prospection, 1998b, 5, pp. 239251 .

ScollAR, Irwin, TABвAGiH, Alain, HI:SSI:, Albert \& HH:Rog, Irmela, Archeological Prospecting and Remote Sensing, Cambridge University Press, 1990.

TABBAGil, Alain, HISSSI:, Albert \& GRARD, Réjean, "Determination of electrical properties of the ground at shallow depth with an electrostatic quadrupole : field trials on archacological sites ", Geophysical Prospecting, 1993, 41, pp. 579-597. 